University of Texas at El Paso

ScholarWorks@UTEP

4-2012

\title{
How to Describe and Propagate Uncertainty When Processing Time Series: Metrological and Computational Challenges, with Potential Applications to Environmental Studies
}

\author{
Christian Servin \\ The University of Texas at El Paso, christians@utep.edu \\ Martine Ceberio \\ The University of Texas at El Paso, mceberio@utep.edu \\ Aline Jaimes \\ The University of Texas at El Paso, ajaimes@utep.edu \\ Craig Tweedie \\ The University of Texas at El Paso, ctweedie@utep.edu \\ Volaldik Kheinovich additional works at: https://scholarworks.utep.edu/cs_techrep \\ The University of Texas at El Paso, vladik@utep.edu \\ Part of the Computer Engineering Commons
}

Comments:

Technical Report: UTEP-CS-12-01a

Published in: Shyi-Ming Chen and and Witold Pedrycz (eds.), Time Series Analysis, Modeling and Applications: A Computational Intelligence Perspective, Springer Verlag, 2013, pp. 279-299.

\section{Recommended Citation}

Servin, Christian; Ceberio, Martine; Jaimes, Aline; Tweedie, Craig; and Kreinovich, Vladik, "How to Describe and Propagate Uncertainty When Processing Time Series: Metrological and Computational Challenges, with Potential Applications to Environmental Studies" (2012). Departmental Technical Reports (CS). 690. https://scholarworks.utep.edu/cs_techrep/690

This Article is brought to you for free and open access by the Computer Science at ScholarWorks@UTEP. It has been accepted for inclusion in Departmental Technical Reports (CS) by an authorized administrator of ScholarWorks@UTEP. For more information, please contact Iweber@utep.edu. 


\title{
How to Describe and Propagate Uncertainty When Processing Time Series: Metrological and Computational Challenges, with Potential Applications to Environmental Studies
}

Christian Servin, Martine Ceberio, Aline Jaimes, Craig Tweedie, and Vladik Kreinovich

\begin{abstract}
Time series comes from measurements, and often, measurement inaccuracy needs to be taken into account, especially in such volatile application areas as meteorology and economics. Traditionally, when we deal with an individual measurement or with a sample of measurement results, we subdivide a measurement error into random and systematic components: systematic error does not change from measurement to measurement while random errors corresponding to different measurements are independent. In time series, when we measure the same quantity at different times, we can also have correlation between measurement errors corresponding to nearby moments of time. To capture this correlation, environmental science researchers proposed to consider the third type of measurement errors: periodic. This extended classification of measurement error may seem ad hoc at first glance, but it leads to a good description of the actual errors. In this paper, we provide a theoretical explanation for this semi-empirical classification, and we show how to efficiently propagate all types of uncertainty via computations.
\end{abstract}

\section{Formulation of the Problem}

In many applications areas - e.g., in meteorology, in financial analysis - the value of the important variable (temperature, stock price, etc.) changes with time. In order to adequately predict the corresponding value, we need to analyze the observed time series and to make a prediction based on this analysis; see, e.g., [3, 20].

All the values that form the time series come from measurements or from expert estimates. Neither measurements nor expert estimates are $100 \%$ accurate, especially in such volatile application areas as meteorology and economics. Thus, the actual values of the corresponding variables are, in general, slightly different from

Cyber-ShARE Center, University of Texas at El Paso, El Paso, TX 79968, USA, contact email e-mail: vladik@utep.edu 
the observed values $x_{t}$. These measurement uncertainties affects the result of data processing.

For example, in meteorological and environmental applications, we measure, at different locations, temperature, humidity, wind speed and direction, flows of carbon dioxide and water between the soil and atmosphere, intensity of the sunlight, reflectivity of the plants, plant surface, etc. Based on these local measurement results, we estimate the regional characteristics such as the carbon fluxes describing the region as a whole - and then use these estimates for predictions. These predictions range from short-term meteorological predictions of weather to short-term environmental predictions of the distribution and survival of different ecosystems and species to long-term predictions of climate change; see, e.g., [1, 12]. Many of these quantities are difficult to measure accurately: for example, the random effects of turbulence and the resulting rapidly changing wind speeds and directions strongly affect our ability to accurately measure carbon dioxide and water flows; see, e.g., [18]. The resulting measurement inaccuracy is one of the main reasons why it is difficult to forecast meteorological, ecological, and climatological phenomena.

It is therefore desirable to describe how the corresponding measurement uncertainty affects the result of data processing. In this paper, we analyze this problem, describe the related challenges, and show how these challenges can be overcome.

\section{Traditional Approach to Measurement Errors}

When we are interested in the value $x$ of some quantity that we can measure directly, we apply an appropriate measuring instrument and get the measurement result $\widetilde{x}$. In the ideal world, the measurement result $\widetilde{x}$ is exactly equal to the desired value $x$. In practice, however, there is noise, there are imperfection, there are other factors which influence the measurement result. As a consequence, the measurement result $\widetilde{x}$ is, in general, different from the actual (unknown) value $x$ of the quantity of interest, and the measurement error $\Delta x \stackrel{\text { def }}{=} \widetilde{x}-x$ is different from 0 .

Because of this, if we repeatedly measure the same quantity by the same measuring instrument, we get, in general, slightly different results. Some of these results are more frequent, some less frequent. For each interval of possible values, we can find the frequency with which the measurement result gets into this interval; at first, some of these frequencies change a lot with each new measurement, but eventually, once we have a large number of measurements, these frequencies stabilize - and become probabilities of different values of $\widetilde{x}$ and, correspondingly, probabilities of different values of measurement error $\Delta x$. In other words, the measurement error becomes a random variable.

Usually, it is assumed that random variables corresponding to different measurement errors are statistically independent from each other. In statistics, independence of two events $A$ and $B$ means that the probability of $A$ does not depend on $B$, i.e., that the conditional probability $P(A \mid B)$ of $A$ under condition $B$ is equal to the unconditional probability $P(A)$ of the event $A$. 
The probability $P(A)$ of the event $A$ can be estimated as the ratio $\frac{N(A)}{N}$ of the number of cases $N(A)$ when the event $A$ occurred to the total number $N$ of observed cases. Similarly, the probability $P(B)$ of the event $B$ can be estimated as the ratio $\frac{N(B)}{N}$ of the number of cases $N(B)$ when the event $A$ occurred to the total number $N$ of observed cases, and the probability $P(A \& B)$ of both events $A$ and $B$ can be estimated as the ratio $\frac{N(A \& B)}{N}$ of the number of cases $N(A \& B)$ when both events $A$ and $B$ occurred to the total number $N$ of observed cases. In contrast, to estimate the conditional probability of $A$ given $B$, we must only take into account cases when $B$ was observed. As a result, we get an estimate $P(A \mid B) \approx \frac{N(A \& B)}{N(B)}$. Since $P(A \& B) \approx \frac{N(A \& B)}{N}$ and $P(B) \approx \frac{N(B)}{N}$, we conclude that $N(A \& B) \approx P(A \& B) \cdot N$ and $N(B) \approx P(B) \cdot N$ and therefore, $P(A \mid B) \approx$ $\frac{P(A \& B) \cdot N}{P(B) \cdot N}=\frac{P(A \& B)}{P(B)}$, so $P(A \mid B) \approx \frac{P(A \& B)}{P(B)}$. The larger the sample, the more accurate are these estimates, so in the limit when $N$ tends to infinity, we get the equality $P(A \mid B)=\frac{P(A \& B)}{P(B)}$, i.e., equivalently, $P(A \& B)=P(A \mid B) \cdot P(B)$. For independent events, $P(A \mid B)=P(A)$ and thus, $P(A \& B)=P(A) \cdot P(B)$.

So, under the independence assumption, if we have two different series of measurements, resulting in measurement errors $\Delta x$ and $\Delta y$, then the probability $P(\Delta x \in[\underline{x}, \bar{x}] \& \Delta y \in[\underline{y}, \bar{y}])$ that $\Delta x$ is in an interval $[\underline{x}, \bar{x}]$ and $\Delta y$ is in an interval $[y, \bar{y}]$ is equal to the product of the two probabilities:

- the probability $P(\Delta x \in[\underline{x}, \bar{x}])$ that $\Delta x$ is in the interval $[\underline{x}, \bar{x}]$, and

- the probability $P(\Delta y \in[y, \bar{y}])$ that $\Delta y$ is in the interval $[y, \bar{y}]$ :

$$
P(\Delta x \in[\underline{x}, \bar{x}] \& \Delta y \in[\underline{y}, \bar{y}])=P(\Delta x \in[\underline{x}, \bar{x}]) \cdot P(\Delta y \in[\underline{y}, \bar{y}]) .
$$

Usually in metrology, the measurement error is divided into two components (see, e.g., [16]):

- the systematic error component, which is defined as the expected value (mean) $E(\Delta x)$ of the measurement errors, and

- the random error component which is defined as the difference $\Delta x-E(\Delta x)$ between the measurement error $\Delta x$ and its systematic component $E(\Delta x)$.

Systematic error component is usually described by the upper bound $\Delta_{s}$ on its absolute value: $|E(\Delta x)| \leq \Delta_{s}$, while the random error is usually described by its mean square value

$$
\sigma=\sqrt{E\left[(\Delta x-E(\Delta x))^{2}\right]}
$$

In statistical terms, $\sigma=\sqrt{V}$ is the standard deviation of the random variable $\Delta x$, i.e., the square root of the variance $V=E\left[(\Delta x-E(\Delta x))^{2}\right]$.

The practical meaning of these components - and the practical difference between them - can be described if, in order to improve measurement accuracy, we repeatedly measure the same quantity several times. Once we have several results 
$\widetilde{x}^{(1)}, \ldots, \widetilde{x}^{(M)}$ of measuring the same (unknown) quantity $x$, we can then take the arithmetic average

$$
\widetilde{x}=\frac{\widetilde{x}^{(1)}+\ldots+\widetilde{x}^{(M)}}{M}
$$

as the new estimate.

One can easily see that the measurement error $\Delta x=\widetilde{x}-x$ corresponding to this new estimate is equal to the average of the measurement errors $\Delta x^{(k)}=\widetilde{x}^{(k)}-x$ corresponding to individual measurements:

$$
\Delta x=\frac{\Delta x^{(1)}+\ldots+\Delta x^{(M)}}{M} .
$$

What are the systematic and random error components of this estimate? Let us start with the systematic error component, i.e., in mathematical terms, with the mean. It is known that the mean of the sum is equal to the sum of the means, and that when we divide a random variable by a constant, its mean is divided by the same constant. All $M$ measurements are performed by the same measuring instrument with the same systematic error $E\left(\Delta x^{(1)}\right)=\ldots=E\left(\Delta x^{(M)}\right)$. Thus, for the $\operatorname{sum} \Delta x^{(1)}+\ldots+\Delta x^{(M)}$, the mean is equal to

$$
E\left(\Delta x^{(1)}+\ldots+\Delta x^{(M)}\right)=E\left(\Delta x^{(1)}\right)+\ldots+E\left(\Delta x^{(M)}\right)=M \cdot E\left(\Delta x^{(k)}\right) .
$$

Therefore, the mean of the ratio $\Delta x$ (which is obtained by dividing the above sum by $M)$ is $M$ times smaller than the mean of the sum, i.e., equal to $E(\Delta x)=E\left(\Delta x^{(k)}\right)$. In other words, the systematic error component does not decrease if we simply repeat the measurements.

In contrast, the random component decreases, or, to be precise, its standard deviation decreases. Indeed, for independent random variables, the variance of the sum is equal to the sum of the variances, and when we divide a random variable by a constant, the variance is divided by the square of this constant. The variance $V=\sigma^{2}$ of each random error component is equal to $V^{(1)}=\ldots=V^{(M)}$; thus, the variance of the sum $\Delta x^{(1)}+\ldots+\Delta x^{(M)}$ is equal to the sum of these variances, i.e., to

$$
V\left[\Delta x^{(1)}+\ldots+\Delta x^{(M)}\right]=V^{(1)}+\ldots+V^{(M)}=M \cdot\left(\sigma^{(k)}\right)^{2} .
$$

Therefore, the variance of the ratio $\Delta x$ (which is obtained by dividing the above sum by $M$ ) is $M^{2}$ times smaller than the variance of the sum, i.e., equal to $\frac{\left(\sigma^{(k)}\right)^{2}}{M}$. So, the standard deviation $\sigma$ (which is the square root of this variance) is equal to $\frac{\sigma^{(k)}}{\sqrt{M}}$. In other words, the more times we repeat the measurement, the smaller the resulting random error. 
So, when we repeat the same measurement several times, the random error disappears, and the only remaining error component is the systematic error.

\section{The Traditional Metrological Approach Does Not Work Well for Time Series}

In the traditional approach, we represent the measurement error as the sum of two components:

- a systematic component which is the same for all measurements, and

- a random component which is independent for different measurements.

When we process time series, this decomposition is insufficient: e.g., usually, there are strong correlations between measurement errors corresponding to consequent measurements.

To achieve a better representation of measurement errors, researchers in environmental science have proposed a semi-empirical idea of introducing the third component of measurement error: the seasonal (periodic) component; see, e.g., [14].

For example, a seasonal error component can represent errors that only happen in spring (this is where the name of this error component comes from), or errors that only happen at night, etc.

From the purely mathematical viewpoint, we can have periodic error components corresponding to all possible frequencies. However, from the physical viewpoint, it makes sense to concentrate on the components with physically meaningful frequencies - and with frequencies which are multiples of these frequencies, e.g., double or triple the daily or yearly frequencies.

For example, in environmental observations, it makes sense to concentrate on daily and yearly periodic errors. If we are interested in the effect of human activity, then we need to add weekly errors - since human activity periodically changes from weekdays to weekends.

The idea of using three components of measurement error works extremely well - which leads to two related challenges:

- A metrological challenge: how can we explain this success? What is the foundation of this idea?

- A computational challenge: how can we efficiently describe this new error component and how can we efficiently propagate it through computations?

In this paper, we address both challenges:

- we provide a theoretical justification for the semi-heuristic idea of the third error component, and

- we show a natural way for efficiently describing this error component, and show how to efficiently propagate different error components through computations. 


\section{First Result: A Theoretical Explanation of the Three-Component Model of Measurement Error}

Our objective is to analyze measurement errors $\Delta x(t)$ corresponding to time series. Namely, we want to represent a generic measurement error as a linear combination of several error components.

This division into components can be described on different levels of granularity. Let us consider the level where the granules are the smallest, i.e., where each granule corresponds to a finite-dimensional linear space, i.e., to the linear space whose elements can be determined by finitely many parameters.

Each component of the measurement error is thus described by a finite-dimensional linear space $L$, i.e., by the set of all the functions of the type $x(t)=c_{1} \cdot x_{1}(t)+\ldots+$ $c_{n} \cdot x_{n}(t)$, where $x_{1}(t), \ldots, x_{n}(t)$ are given functions, and $c_{1}, \ldots, c_{n}$ are arbitrary constants.

In most applications, observed signals continuously (and even smoothly) depend on time, so we will assume that all the functions $x_{i}(t)$ are smooth (differentiable).

Also, usually, there is an upper bound on the measurement error, so we will assume that each of the the functions $x_{i}(t)$ are bounded by a constant.

Finally, for a long series of observations, we can choose a starting point arbitrarily. If instead of the original starting point, we take a starting point which is $t_{0}$ seconds earlier, then each moment of time which was originally described as moment $t$ is not described as moment $t+t_{0}$. Then, for describing measurement errors, instead of the original function $x(t)$, we have a new function $x_{t_{0}}(t)$ for which $x_{t_{0}}\left(t+t_{0}\right)=x\left(t+t_{0}\right)$. It is reasonable to require that the linear space that describes a component of the measurement error does not not change simply because we changed the starting point. Thus, we arrive at the following definitions.

Definition 1. We say that a function $x(t)$ of one variable is bounded if there exists a constant $M$ for which $|x(t)| \leq M$ for all $t$.

Definition 2. We say that a class $F$ of functions of one variable is shift-invariant if for every function $x(t) \in F$ and for every real number $t_{0}$, the function $x\left(t+t_{0}\right)$ also belongs to the class $F$.

Definition 3. By an error component we mean a shift-invariant finite-dimensional linear space of functions

$$
L=\left\{c_{1} \cdot x_{1}(t)+\ldots+c_{n} \cdot x_{n}(t)\right\}
$$

where $x_{1}(t), \ldots, x_{n}(t)$ are given bounded smooth functions and $c_{i}$ are arbitrary numbers.

Theorem 1. Every error component is a linear combination of the functions

$$
x(t)=\sin (\omega \cdot t) \text { and } x(t)=\cos (\omega \cdot t) .
$$


Proof.

$1^{\circ}$. Let us first use the assumption that the linear space $L$ is shift-invariant.

For every $i$ from 1 to $n$, the corresponding function $x_{i}(t)$ belongs to the linear space $L$. Since the error component is shift-invariant, we can conclude that for every real number $t_{0}$, the function $x_{i}\left(t+t_{0}\right)$ also belongs to the same linear space. Thus, for every $i$ from 1 to $n$ and for every $t_{0}$, there exist values $c_{1}, \ldots, c_{n}$ (possibly depending on $i$ and on $t_{0}$ ) for which

$$
x_{i}\left(t+t_{0}\right)=c_{i 1}\left(t_{0}\right) \cdot x_{1}(t)+\ldots+c_{i n}\left(t_{0}\right) \cdot x_{n}(t)
$$

$2^{\circ}$. We know that the functions $x_{1}(t), \ldots, x_{n}(t)$ are smooth. Let us use the equation (1) to prove that the functions $c_{i j}\left(t_{0}\right)$ are also smooth (differentiable).

Indeed, if we substitute $n$ different values $t_{1}, \ldots, t_{n}$ into the equation (1), then we get a system of $n$ linear equations with $n$ unknowns to determine $n$ values $c_{i 1}\left(t_{0}\right)$, $\ldots, c_{\text {in }}\left(t_{0}\right)$ :

$$
\begin{gathered}
x_{i}\left(t_{1}+t_{0}\right)=c_{i 1}\left(t_{0}\right) \cdot x_{1}\left(t_{1}\right)+\ldots+c_{i n}\left(t_{0}\right) \cdot x_{n}\left(t_{1}\right) ; \\
\ldots \\
x_{i}\left(t_{n}+t_{0}\right)=c_{i 1}\left(t_{0}\right) \cdot x_{1}\left(t_{n}\right)+\ldots+c_{i n}\left(t_{0}\right) \cdot x_{n}\left(t_{n}\right) .
\end{gathered}
$$

The solution of a system of linear equations - as determined by the Cramer's rule is a smooth function of all the coefficients and right-hand sides. Since all the righthand sides $x_{i}\left(t_{j}+t_{0}\right)$ are smooth functions of $t_{0}$ and since all the coefficients $x_{i}\left(t_{j}\right)$ are constants (and thus, are also smooth), we conclude that each dependence $c_{i j}\left(t_{0}\right)$ is indeed smooth.

$3^{\circ}$. Now that we know that all the functions $x_{i}(t)$ and $c_{i j}\left(t_{0}\right)$ are differentiable, we can differentiate both sides of the equation (1) with respect to $t_{0}$ and then take $t_{0}=0$. As a result, we get the following systems of $n$ differential equations with $n$ unknown functions $x_{1}(t), \ldots, x_{n}(t)$ :

$$
\dot{x}_{i}(t)=c_{i 1} \cdot x_{1}(t)+\ldots+c_{i n} \cdot x_{n}(t),
$$

where $\dot{x}_{i}(t)$ denotes derivative over time, and $c_{i j}$ denoted the value of the corresponding derivative $\dot{c}_{i j}$ when $t_{0}=0$.

$3^{\circ}$. We have shown that the functions $x_{1}(t), \ldots, x_{n}(t)$ satisfy a system of linear differential equations with constant coefficients.

It is known that a general solution of such system of equations is a linear combination of functions of the type $t^{k} \cdot \exp (\lambda \cdot t)$, where $k$ is a natural number (nonnegative integer), and $\lambda$ is a complex number. Specifically, $\lambda$ is an eigenvalue of the matrix $c_{i j}$, and the value $k>0$ appears when we have a degenerate eigenvalue, i.e., an eigenvalue for which there are several linearly independent eigenvectors.

$4^{\circ}$. Every complex number $\lambda$ has the form $a+\mathrm{i} \cdot \omega$, where $a$ is its real part and $\omega$ is its imaginary part. So: 


$$
\exp (\lambda \cdot t)=\exp (a \cdot t) \cdot \cos (\omega \cdot t)+\mathrm{i} \cdot \exp (a \cdot t) \cdot \sin (\omega \cdot t)
$$

Thus, every function $x_{i}(t)$ can be represented as a linear combination of expressions of the types $t^{k} \cdot \exp (a \cdot t) \cdot \cos (\omega \cdot t)$ and $t^{k} \cdot \exp (a \cdot t) \cdot \sin (\omega \cdot t)$.

$5^{\circ}$. Now, we can use the requirement that the functions $x_{i}(t)$ are bounded.

$5.1^{\circ}$. Because of this requirement, we cannot have $a \neq 0$ :

- for $a>0$, the function is unbounded for $t \rightarrow+\infty$, while

- for $a<0$, the function is unbounded for $t \rightarrow-\infty$.

So, we must have $a=0$.

$5.2^{\circ}$. Similarly, if $k>0$, the corresponding function is unbounded. Thus, we must have $k=0$.

$6^{\circ}$. Thus, every function $x_{i}(t)$ is a linear combination of the trigonometric functions $x(t)=\sin (\omega \cdot t)$ and $x(t)=\cos (\omega \cdot t)$.

The theorem is proven.

What are the practical conclusions of this result? We have concluded that the measurement error $\Delta x(t)$ can be described as a linear combination of sines and cosines corresponding to different frequencies $\omega$.

In practice, depending on the relation between the frequency $\omega$ and the frequency $f$ with which we perform measurements, we can distinguish between small, medium, and large frequencies:

- frequencies $\omega$ for which $\omega \ll f$ are small;

- frequencies $\omega$ for which $\omega \gg f$ are large, and

- all other frequencies $\omega$ are medium.

Let us consider these three types of frequencies one by one.

When the frequency $\omega$ is low, the corresponding values $\cos (\omega \cdot t)$ and $\sin (\omega \cdot t)$ practically do not change with time: the change period is much larger than the usual observation period.

Thus, we can identify low-frequency components with systematic error component - the error component that practically does not change with time.

When the frequency $\omega$ is high, $\omega \gg f$, the phases of the values $\cos \left(\omega \cdot t_{i}\right)$ and $\cos \left(\omega \cdot t_{i+1}\right)$ (or, alternatively,

$\sin \left(\omega \cdot t_{i}\right)$ and $\left.\sin \left(\omega \cdot t_{i+1}\right)\right)$ corresponding to the two sequential measurements $t_{i}$ and $t_{i+1}$ differ so much that for all practical purposes, the resulting values of cosine or sine functions are independent.

Thus, high-frequency components can be identified with random error component - the error component for which measurement errors corresponding to different measurements are independent.

In contrast to the cases of low and high frequencies, where the periodicity of the corresponding cosine and sine functions is difficult to observe, components $\cos (\omega$. $t)$ and $\sin (\omega \cdot t)$ corresponding to medium frequencies $\omega$ are observably periodic. 
It is therefore reasonable to identify medium-frequency error components with seasonal (periodic) components of the measurement error.

This conclusion explains why, in addition to the original physically meaningful frequencies, it is also reasonable to consider their multiples:

- We know that the corresponding error component is a periodic function of time, with the physically meaningful period $T_{0}$.

- It is known that every periodic function can be explained into Fourier series, i.e., represented as a linear combination of sines and cosines with frequencies $\omega$ which are multiples of the basic frequency $\omega_{0}=\frac{2 \pi}{T_{0}}$ corresponding to the period $T_{0}$.

Thus, we have indeed provided a justification to the semi-empirical three-component model of measurement error.

\section{Periodic Error Component: Technical Details}

In the above section, we explained that the periodic error component is as fundamental as the more traditional systematic and random error components. It is therefore necessary to extend the usual analysis of error components and their propagation to this new type of measurement errors.

For systematic and random error components we know:

- how to describe reasonable bounds on this error component, and

- how to estimate this error component when we calibrate the measuring instrument.

Specifically, the random error component is characterized by its standard deviation $\sigma$, while a systematic error component $s$ is characterized by the upper bound $\Delta$ : $|s| \leq \Delta$.

The standard deviation $\sigma$ of the measuring instrument can be estimated if we repeatedly measure the same quantity $x$ by this instrument. Then, the desired standard deviation can be estimated as the sample standard deviation of the corresponding measurement results $\widetilde{x}^{(1)}, \ldots, \widetilde{x}^{(M)}$ :

$$
\sigma \approx \sqrt{\frac{1}{M} \cdot \sum_{k=1}^{M}\left(\widetilde{x}^{(k)}-E\right)^{2}},
$$

where $E=\frac{1}{M} \cdot \sum_{k=1}^{M} \widetilde{x}^{(k)}$.

To estimate the systematic error component, it is not enough to have the given measuring instrument, we also need to calibrate the measuring instrument, i.e., to measure the same quantity $x$ with an additional much more accurate ("standard") measuring instrument - whose measurement result $\widetilde{x}_{s}$ is assumed to be very close 
to the actual value $x$ of the measured quantity. Here, $E \approx E(\widetilde{x})$ and $\widetilde{x}_{s} \approx x$, so the difference $E-x_{s}$ is approximately equal to $E(\widetilde{x})-x=E(\tilde{x}-x)=E(\Delta x)$. Thus, this difference $E-\widetilde{x}_{s}$ can be used as a good approximation to the systematic error component.

Since we want to also take into account the periodic error component, it is desirable to provide answers to the above two questions for the periodic error component as well.

How can we describe reasonable bounds for each part of the periodic error component? For each frequency $\omega$, the corresponding linear combination

$$
a_{c} \cdot \cos (\omega \cdot t)+a_{s} \cdot \sin (\omega \cdot t)
$$

can be equivalently represented as $A \cdot \cos (\omega \cdot t+\varphi)$. This is the form that we will use for describing the periodic error component.

Similarly to the systematic error, for the amplitude $A$, we will assume that we know the upper bound $P:|A| \leq P$.

For phase $\varphi$, it is natural to impose a requirement that the probability distribution of phase be invariant with respect to shift $t \rightarrow t+t_{0}$. When time is thus shifted, the phase is also shifted by $\varphi_{0}=\omega \cdot t_{0}$. Thus, the requirement leads to the conclusion that the probability distribution for the phase be shift-invariant, i.e., that the corresponding probability density function $\rho(\varphi)$ is shift-invariant $\rho(\varphi)=\rho\left(\varphi+\varphi_{0}\right)$ for every possible shift $\varphi_{0}$. This means that this probability density function must be constant, i.e., that the phase $\varphi$ is uniformly distributed on the interval $[0,2 \pi]$.

How can we estimate the periodic error component when calibrating a measuring instrument? When we compare the results of measuring the time series by our measuring instrument and by a standard measuring instrument, we get a sequence of differences $\widetilde{x}(t)-\widetilde{x}_{s}(t)$ that approximates the actual measurement errors $\Delta x(t)$.

Periodic error components are sinusoidal components corresponding to several frequencies. In data processing, there is a known procedure for representing each sequence as a linear combination of sinusoids of different frequency - Fourier transform. To find the periodic components, it is therefore reasonable to perform a Fourier Transform; the amplitudes of the Fourier transform corresponding to physically meaningful frequencies (and their multiples) $\omega$ will then serve as estimates for the amplitude of the corresponding periodic measurement error component.

Computing Fourier transform is fast: there is a known Fact Fourier Transform (FFT) algorithm for this computation; see, e.g., [2].

In this process, there is a still a computational challenge. Indeed, while the standard measuring instrument is reasonably accurate and its measurement results $\widetilde{x}_{s}(t)$ provide a good approximation to the actual values $x(t)$, these results are still somewhat different from the actual values $x(t)$. Hence, the observed differences $\widetilde{x}(t)-\widetilde{x}_{s}(t)$ are only approximately equal to the measurement errors $\Delta x(t)=$ $\widetilde{x}(t)-x(t)$. When we apply FFT in a straightforward way, this approximation error sometimes leads to drastic over-estimation of the results; see, e.g., [4, 13]. Because of this fact, many researchers replaced FFT by much slower - but more accurate error estimation algorithms. 
In our paper [13], we showed how we can modify the FFT techniques so that we get (almost) exact error bounds while being (almost) as fast as the original FFT. So, to estimate the periodic error component, we need to use thus modified FFT algorithm.

\section{Because of Our Justification, the Three-Component Model of Approximation Error Can Also Be Applied to Expert Estimates}

In many practical situations, the measurement results are not sufficient to make reasonable conclusions. We need to supplement measurement results with the knowledge of experts. The use of expert knowledge in processing data is one of the important aspects of computational intelligence.

For example, when a medical doctor makes a diagnosis and/or prescribes medicine, he or she is usually not following an algorithm that inputs the patients stats and outputs the name of the disease and the dosage of the corresponding medicine. If medicine was that straightforward, there would have been no need for skilled medical doctors. A good doctor also uses his/her experience, his/her intuition. Similarly, in environmental research, we measure temperature, humidity, etc. However, to make meaningful conclusions, it is necessary to supplement these measurement results with expert estimates of, e.g., amount of leaves on the bushes ("low", "medium", "high"), state of the leaves - and many other characteristics which are difficult to measure but which can be easily estimated by an expert.

We have mentioned that in data processing, it is important to take into account the uncertainty of measurement results. Expert estimates are usually even much less accurate than measurement results. So, it is even more important to take into account the uncertainty of expert estimates.

The main idea behind most methods for dealing with uncertainty of expert estimates is to treat an expert as a measuring instrument and use the corresponding metrological techniques.

One of the main techniques for describing expert uncertainty is fuzzy techniques; see, e.g., $[9,15]$. While these techniques are not exactly probabilistic, many fuzzy techniques are similar to the probabilistic ones.

For example, one of the most widely used methods of determining the (fuzzy) degree of belief $\mu_{P}(x)$ that a certain value $x$ satisfies the property $P$ (e.g., that a certain temperature is low) is to poll several experts and take, as $\mu_{P}(x)$, the proportion of those who thing that $x$ satisfies this property.

Good news is that in our analysis of the error components, we never used the fact that this error comes from measurements. We can therefore apply the exact same analysis to the approximation error of the expert estimates.

Thus, while our main current emphasis is on measurement results and measurement uncertainty, it is desirable to apply the same three-component decomposition to inaccuracies of expert estimates as well. 


\section{How to Propagate Uncertainty in the Three-Component Model}

In the previous sections, we analyzed how to describe the uncertainty related to measurements and/or expert estimates. Some quantities can be indeed directly measured or estimates. However, there are many quantities of interest which cannot be directly measured or estimated.

An example of such a quantity is a carbon flux that describes the exchange of carbon between the soil and the atmosphere; see, e.g., [12]. It is difficult to measure this flux directly. Instead, we measure the humidity, wind and concentration of different gases at different height of a special meteorological tower, and then use the results of these measurements to process the data.

In general, for many quantities $y$, it is not easy (or even impossible) to measure them directly. Instead, we measure related quantities $x_{1}, \ldots, x_{n}$, and use the known relation $y=f\left(x_{1}, \ldots, x_{n}\right)$ between $x_{i}$ and $y$ to estimate the desired quantity $y$.

Since measurements come with uncertainty, the resulting estimate is, in general, somewhat different from the actual value of the desired quantity - even when the relation $y=f\left(x_{1}, \ldots, x_{n}\right)$ is known exactly. It is therefore desirable to propagate this uncertainty, i.e., to find out how accurate is the estimate based on (approximate) measurement results.

In practical applications, many inputs to the data processing algorithm come from the same sensor at different moments of time. In other words, as inputs, we have the results $\widetilde{x}_{i}\left(t_{i j}\right)$ of measuring the values $x_{i}\left(t_{i j}\right)$ by the $i$-th sensor at the $j$-th moment of time $t_{i j}=t_{0}+j \cdot \Delta t_{i}$, where $t_{0}$ is the starting moment of all the measurements, and $\Delta t_{i}$ is the time interval between the two consecutive measurements performed by the $i$-th sensor.

The desired quantity $y$ depends on all these values:

$$
y=f\left(x_{1}\left(t_{11}\right), x_{1}\left(t_{12}\right), \ldots, x_{2}\left(t_{21}\right), x_{2}\left(t_{22}\right), \ldots, x_{n}\left(t_{n 1}\right), x_{n}\left(t_{n 2}\right), \ldots\right) .
$$

Instead of the actual values $x_{i}\left(t_{i j}\right.$, we only know the measurement results $\widetilde{x}_{i}\left(t_{i j}\right)$, results which differ from the actual values by the corresponding measurement errors $\Delta x_{i}\left(t_{i j}\right)$ :

$$
\widetilde{x}_{i}\left(t_{i j}\right)=x_{i}\left(t_{i j}\right)+\Delta x_{i}\left(t_{i j}\right) .
$$

After applying the data processing algorithm $f$ to the measurement results $\widetilde{x}_{i}\left(t_{i j}\right)$, we get the estimate $\tilde{y}$ for the desired quantity $y$ :

$$
\widetilde{y}=f\left(\widetilde{x}_{1}\left(t_{11}\right), \widetilde{x}_{1}\left(t_{12}\right), \ldots, \widetilde{x}_{n}\left(t_{n 1}\right), \widetilde{x}_{n}\left(t_{n 2}\right), \ldots\right) .
$$

We are interested in estimating the difference

$$
\begin{gathered}
\Delta y=\tilde{y}-y=f\left(\widetilde{x}_{1}\left(t_{11}\right), \widetilde{x}_{1}\left(t_{12}\right), \ldots, \widetilde{x}_{n}\left(t_{n 1}\right), \widetilde{x}_{n}\left(t_{n 2}\right), \ldots\right)- \\
f\left(x_{1}\left(t_{11}\right), x_{1}\left(t_{12}\right), \ldots, x_{n}\left(t_{n 1}\right), x_{n}\left(t_{n 2}\right), \ldots\right) .
\end{gathered}
$$

We know that the actual (unknown) value $x_{i}\left(t_{i j}\right)$ of each measured quantity is equal to 


$$
x_{i}\left(t_{i j}\right)=\widetilde{x}_{i}\left(t_{i j}\right)-\Delta x_{i}\left(t_{i j}\right) .
$$

Thus, the desired difference has the form

$$
\begin{gathered}
\Delta y=f\left(\widetilde{x}_{1}\left(t_{11}\right), \ldots, \widetilde{x}_{n}\left(t_{n 1}\right), \widetilde{x}_{n}\left(t_{n 2}\right), \ldots\right)- \\
f\left(\widetilde{x}_{1}\left(t_{11}\right)-\Delta x_{1}\left(t_{11}\right), \ldots, \widetilde{x}_{n}\left(t_{n 1}\right)-\Delta x_{n}\left(t_{n 1}\right), \widetilde{x}_{n}\left(t_{n 2}\right)-\Delta x_{n}\left(t_{n 2}\right), \ldots\right) .
\end{gathered}
$$

Our objective is to estimate this difference based on the known information about the measurement errors $\Delta x_{i}\left(t_{i j}\right)$.

Measurement errors are usually relatively small, so terms quadratic and of higher order in terms of $\Delta x_{i}\left(t_{i j}\right)$ can be safely ignored.

For example, if the measurement error is $10 \%$, its square is $1 \%$ which is much much smaller than $10 \%$. If we measure with a higher accuracy, e.g., of $1 \%$, then the square of this value is $0.01 \%$ which is even mich more smaller than the error itself.

Thus, we can linearize the above formula, i.e., expand the dependence of $\Delta y$ on $\Delta x_{i}\left(t_{i j}\right)$ in Taylor series and keep only linear terms in this expansion. As a result, we arrive at the following formula:

$$
\Delta y=\sum_{i} \sum_{j} C_{i j} \cdot \Delta x_{i}\left(t_{i j}\right)
$$

where $C_{i j}$ denotes the corresponding partial derivative $\frac{\partial y}{\partial x_{i}\left(t_{i j}\right)}$.

As a result of this linearization, we can consider all three components separately. Indeed, we know that each measurement errors $\Delta x_{i}\left(t_{i j}\right)$ consists of three components: systematic component $s_{i}$, random component $r_{i j}$, and periodic component(s) $A_{\ell i} \cdot \cos \left(\omega_{\ell} \cdot t_{i j}+\varphi_{\ell i}\right)$ corresponding to different physically meaningful frequencies (and their multiples) $\omega_{\ell}$ :

$$
\Delta x_{i}\left(t_{i j}\right)=s_{i}+r_{i j}+\sum_{\ell} A_{\ell i} \cdot \cos \left(\omega_{\ell} \cdot t_{i j}+\varphi_{\ell i}\right) .
$$

The dependence of $\Delta y$ on the measurement errors $\Delta x_{i}\left(t_{i j}\right)$ is linear. Thus, we can represent $\Delta y$ as the sum of different components coming from, correspondingly, systematic, random, and periodic errors:

$$
\Delta y=\Delta y_{s}+\Delta y_{r}+\sum_{\ell} \Delta y_{p \ell},
$$

where

$$
\begin{gathered}
\Delta y_{s}=\sum_{i} \sum_{j} C_{i j} \cdot s_{i} ; \\
\Delta y_{r}=\sum_{i} \sum_{j} C_{i j} \cdot r_{i j} \\
\Delta y_{p \ell}=\sum_{i} \sum_{j} C_{i j} \cdot A_{\ell i} \cdot \cos \left(\omega_{\ell} \cdot t_{i j}+\varphi_{\ell i}\right) .
\end{gathered}
$$


So, it is indeed sufficient to estimate the effect of all three types of measurement error components separately.

In these estimations, we will make a natural assumption: that measurement errors corresponding to different time series are independent. Indeed, as we have mentioned earlier,

- while measurement errors corresponding to measurement by the same sensor at consecutive moments of time are correlated,

- measurement errors corresponding to different sensors usually come from different factors and are, therefore, largely independent.

Because of this assumption, we arrive at the following algorithms for estimating different components of $\Delta y$.

Propagating random component is the traditional part of error propagation. A natural way to describe the resulting error $\Delta y_{r}$ is to use simulations (i.e., a so-called Monte-Carlo approach).

By definition of the random error component, the values $r_{i j}$ and $r_{i k}$ corresponding to measurements by the same $i$-th sensor at different moments of time $t_{i j}$ and $t_{i j^{\prime}}$ are independent. We are also assuming that the values $r_{i j}$ and $r_{i^{\prime} j^{\prime}}$ corresponding to different sensors are independent. Thus, all the values $r_{i j}$ corresponding to different pairs $(i, j)$ are independent.

There are many such values, since each sensor performs the measurements with a high frequency - e.g., one reading every second or every minute. The value $\Delta y_{r}$ is thus a linear combination of a large number of independent random variables. It is known that under reasonable conditions, the probability distribution of such a combination tends to normal; this is what is known as the Central Limit Theorem - one of the main reasons why normal distributions are ubiquitous in nature; see, e.g., [19].

A normal distribution is uniquely determined by its mean and standard deviation. We know that each measurement error $r_{i j}$ has mean 0 and a known standard deviation $\sigma_{i}$ corresponding to measurements of the $i$-th sensor. The mean of the linear combination is equal to the linear combination of means. Thus, the mean of $\Delta y_{r}$ is 0 . The standard deviation can be obtained if we repeatedly simulate random errors and take a standard deviation of the corresponding empirical values $\Delta y_{r}^{(1)}, \Delta y_{r}^{(2)}$, ... Thus, we arrive at the following algorithm.

Propagating random component: algorithm. The random component $\Delta y_{r}$ is normally distributed with zero mean. Its standard deviation can be obtained as follows:

- First, we apply the algorithm $f$ to the measurement results $\tilde{x}_{i}\left(t_{i j}\right)$ and get the estimate $\tilde{y}$.

- Then, for $k=1, \ldots, N$, we do the following:

- simulate the random errors $r_{i j}^{(k)}$ as independent random variables (e.g., Gaussian) with 0 mean and standard deviation $\sigma_{i}$;

- form simulated values $x_{i}^{(k)}\left(t_{i j}\right)=\widetilde{x}_{i}\left(t_{i j}\right)-r_{i j}^{(k)}$; 
- substitute the simulated values $x_{i}^{(k)}\left(t_{i j}\right)$ into the data processing algorithm $f$ and get the result $y^{(k)}$.

- Finally, we estimate the standard deviation $\sigma$ of the random component $\Delta y_{r}$ as

$$
\sigma=\sqrt{\frac{1}{N} \cdot \sum_{k=1}^{N}\left(y^{(k)}-\widetilde{y}\right)^{2}}
$$

Mathematical comment. The proof that this algorithm produces a correct result easily follows from the fact that for simulated values, the difference $y^{(k)}-\widetilde{y}$ has the form $\sum_{i} \sum_{j} C_{i j} \cdot r_{i j}^{(k)}$ and thus, has the exact same distribution as $\Delta y_{r}=\sum_{i} \sum_{j} C_{i j} \cdot \Delta x_{i}\left(t_{i j}\right)$; see, e.g., [10].

Metrological comment. In some practical situations, instead of the standard deviations $\sigma_{i}=\sqrt{E\left[(\Delta x)^{2}\right]}$ that describe the absolute accuracy, practitioners often describe relative accuracy $\delta_{i}$ such as $5 \%$ or $10 \%$. In this case, the standard deviation $\sigma_{i}$ can be obtained as $\sigma_{i}=\delta_{i} \cdot m_{i}$, i.e., by multiplying the given value $\delta_{i}$ and the mean square value of the signal

$$
m_{i}=\sqrt{\frac{1}{T_{i}} \cdot \sum_{j}\left(\widetilde{x}_{i}\left(t_{i j}\right)\right)^{2}},
$$

where $T_{i}$ is the total number of measurements performed by the $i$-th sensor.

Let us now consider the problem of propagating systematic component. By definition, the systematic component $\Delta y_{s}$ of the resulting error $\Delta y$ is equal to $\Delta y_{s}=\sum_{i} \sum_{j} C_{i j} \cdot s_{i}$. If we combine terms corresponding to different $j$, we conclude that $\Delta y_{s}=\sum_{i} K_{i} \cdot s_{i}$, where $K_{i} \stackrel{\text { def }}{=} \sum_{j} C_{i j}$.

The values $K_{i}$ can be explicitly described. Namely, one can easily see that if for some small value $\delta>0$, for this sensor $i$, we take $\Delta x_{i}\left(t_{i j}\right)=\delta$ for all $j$, and for all other sensors $i^{\prime}$, we take $\Delta x_{i^{\prime}}\left(t_{i^{\prime} j}\right)=0$, then the resulting increase in $y$ will be exactly equal to $\delta \cdot K_{i}$.

Once we have determined the coefficients $K_{i}$, we need to find out the smallest and the largest possible value of the sum $\Delta y_{s}=\sum_{i} K_{i} \cdot s_{i}$. Each parameter $s_{i}$ can take any value between $-\Delta_{s i}$ and $\Delta_{s i}$, and these parameters are independent. Thus, the sum is the largest when each term $K_{i} \cdot s_{i}$ is the largest.

Each term is a linear function of $s_{i}$. A linear function is increasing or decreasing depending on whether the coefficient $K_{i}$ is positive or negative.

- When $K_{i} \geq 0$, the linear function $K_{i} \cdot s_{i}$ is increasing and thus, its largest possible value is attained when $s_{i}$ attains its largest possible value $\Delta_{s i}$. Thus, this largest possible value is equal to $K_{i} \cdot \Delta_{s i}$. 
- When $K_{i} \leq 0$, the linear function $K_{i} \cdot s_{i}$ is decreasing and thus, its largest possible value is attained when $s_{i}$ attains its smallest possible value $-\Delta_{s i}$. Thus, this largest possible value is equal to $-K_{i} \cdot \Delta_{s i}$.

In both cases, the largest possible value is equal to $\left|K_{i}\right| \cdot \Delta_{s i}$ and thus, the largest possible value $\Delta_{s}$ of the sum $\Delta y_{s}$ is equal to $\Delta_{s} \stackrel{\text { def }}{=} \sum_{i}\left|K_{i}\right| \cdot \Delta_{s i}$. Similarly, one can prove that the smallest possible value of $\Delta y_{s}$ is equal to $-\Delta_{s}$.

Thus, we arrive at the following algorithm for computing the upper bound $\Delta_{s}$ on the systematic component $\Delta y_{s}$.

Propagating systematic component: algorithm. The largest possible value $\Delta_{s}$ of the systematic component $\Delta y_{s}$ can be obtained as follows:

- First, we apply the algorithm $f$ to the measurement results $\widetilde{x}_{i}\left(t_{i j}\right)$ and get the estimate $\tilde{y}$.

- Then, we select a small value $\delta$ and for each sensor $i$, we do the following:

- for this sensor $i$, we take $x_{i}^{(i)}\left(t_{i j}\right)=\widetilde{x}_{i}\left(t_{i j}\right)+\delta$ for all moments $j$;

- for all other sensors $i^{\prime} \neq i$, we take $x_{i^{\prime}}^{(i)}\left(t_{i^{\prime} j}\right)=\widetilde{x}_{i}\left(t_{i^{\prime} j}\right)$;

- substitute the resulting values $x_{i^{\prime}}^{(i)}\left(t_{i^{\prime} j}\right)$ into the data processing algorithm $f$ and get the result $y^{(i)}$.

- Finally, we estimate the desired bound $\Delta_{s}$ on the systematic component $\Delta y_{s}$ as

$$
\Delta_{s}=\sum_{i}\left|\frac{y^{(i)}-\tilde{y}}{\delta}\right| \cdot \Delta_{s i}
$$

Metrological comment. In some practical situations, instead of the absolute bound $\Delta_{s i}$ on the systematic error of the $i$-th sensor, practitioners often describe relative accuracy $\delta_{i}$ such as $5 \%$ or $10 \%$. In this case, a reasonable way to describe the absolute bound is to determine it as $\Delta_{s i}=\delta_{i} \cdot a_{i}$, i.e., by multiplying the given value $\delta_{i}$ and the mean absolute value of the signal

$$
a_{i}=\frac{1}{T_{i}} \cdot \sum_{j}\left|\widetilde{x}_{i}\left(t_{i j}\right)\right|
$$

Numerical example. Let us consider a simple case when we are estimating the difference between the average temperatures at two nearby locations. For example, we may be estimating the effect of a tree canopy on soil temperature, by comparing the temperature at a forest location with the temperature at a nearby clearance location. Alternatively, we can be estimating the effect of elevation of the temperature by comparing the temperatures at different elevations. In this case, we use the same frequency $\Delta t_{1}=\Delta t_{2}$ for both sensors, so $t_{1 j}=t_{2 j}=t_{j}$. The difference in average temperatures is defined as 


$$
\begin{gathered}
y=f\left(x_{1}\left(t_{0}\right), x_{2}\left(t_{0}\right), x_{1}\left(t_{1}\right), \ldots, x_{2}\left(t_{1}\right), \ldots, x_{1}\left(t_{n}\right), x_{2}\left(t_{n}\right)\right)= \\
\frac{x_{1}\left(t_{0}\right)+\ldots+x_{1}\left(t_{n}\right)}{n+1}-\frac{x_{2}\left(t_{0}\right)+\ldots+x_{2}\left(t_{n}\right)}{n+1} .
\end{gathered}
$$

Let us assume that the know upper bound on the systematic error of the first sensor is $\Delta_{s 1}=0.1$, and the upper bound on the systematic error of the second sensor is $\Delta_{s 2}=0.2$. We perform measurements at three moments of time $t=0,1,2$. During these three moments of time, the first sensor measured temperatures $\widetilde{x}_{1}\left(t_{0}\right)=20.0$, $\tilde{x}_{1}\left(t_{1}\right)=21.9$, and $\tilde{x}_{1}\left(t_{2}\right)=18.7$, and the second second measured temperatures $\widetilde{x}_{2}\left(t_{0}\right)=22.4, \widetilde{x}_{2}\left(t_{1}\right)=23.5$, and $\widetilde{x}_{2}\left(t_{2}\right)=21.0$. In this case, the estimate $\widetilde{y}$ for the desired difference $y$ between average temperatures is equal to

$$
\tilde{y}=\frac{20.0+21.9+18.7}{3}-\frac{22.4+23.5+21.0}{3}=20.2-22.3=-2.1 \text {. }
$$

According to our algorithm, we first select a small value $\delta$, e.g., $\delta=0.1$.

Then, we modify the results of the first sensor while keeping the results of the second sensor unchanged. As a result, we get $x_{1}^{(1)}\left(t_{0}\right)=\widetilde{x}_{1}\left(t_{0}\right)+\delta=20.0+0.1=$ 20.1 , and similarly $x_{1}^{(1)}\left(t_{1}\right)=22.0$ and $x_{1}^{(1)}\left(t_{2}\right)=18.8$; we also get $x_{2}^{(1)}\left(t_{0}\right)=\widetilde{x}_{2}\left(t_{0}\right)=$ 22.4 , and similarly $x_{2}^{(1)}\left(t_{1}\right)=23.5$ and $x_{2}^{(1)}\left(t_{2}\right)=21.0$. For thus modified values, we get

$$
\begin{gathered}
y^{(1)}=\frac{x_{1}^{(1)}\left(t_{0}\right)+x_{1}^{(1)}\left(t_{1}\right)+x_{1}^{(1)}\left(t_{2}\right)}{3}-\frac{x_{2}^{(1)}\left(t_{0}\right)+x_{2}^{(1)}\left(t_{1}\right)+x_{2}^{(1)}\left(t_{2}\right)}{3}= \\
\frac{20.1+22.0+18.8}{3}-\frac{22.3+23.5+21.0}{3}=20.3-22.3=-2.0 .
\end{gathered}
$$

Similarly, we modify the results of the second sensor while keeping the results of the first sensor unchanged. As a result, we get $x_{1}^{(2)}\left(t_{0}\right)=\widetilde{x}_{1}\left(t_{0}\right)=20.0$, and similarly $x_{1}^{(2)}\left(t_{1}\right)=21.9$ and $x_{1}^{(2)}\left(t_{2}\right)=18.7$; we also get $x_{2}^{(2)}\left(t_{0}\right)=\widetilde{x}_{2}\left(t_{0}\right)+\delta=22.4+0.1=$ 22.5 , and similarly $x_{2}^{(2)}\left(t_{1}\right)=23.6$ and $x_{2}^{(2)}\left(t_{2}\right)=21.1$. For thus modified values, we get

$$
\begin{gathered}
y^{(2)}=\frac{x_{1}^{(2)}\left(t_{0}\right)+x_{1}^{(2)}\left(t_{1}\right)+x_{1}^{(2)}\left(t_{2}\right)}{3}-\frac{x_{2}^{(2)}\left(t_{0}\right)+x_{2}^{(2)}\left(t_{1}\right)+x_{2}^{(2)}\left(t_{2}\right)}{3}= \\
\frac{20.0+21.9+18.7}{3}-\frac{22.4+23.6+21.1}{3}=20.2-22.4=-2.2 .
\end{gathered}
$$

Finally, we estimate the desired bound $\Delta_{s}$ on the systematic component $\Delta_{s} y$ as

$$
\begin{gathered}
\Delta_{s}=\frac{\left|y^{(1)}-\tilde{y}\right|}{\delta} \cdot \Delta_{s 1}+\frac{\left|y^{(2)}-\tilde{y}\right|}{\delta} \cdot \Delta_{s 2}= \\
\frac{|(-2.0)-(-2.1)|}{0.1} \cdot 0.1+\frac{|(-2.2)-(-2.1)|}{0.1} \cdot 0.3=1 \cdot 0.1+1 \cdot 0.3=0.4 .
\end{gathered}
$$


Finally, let us consider the problem of propagating the periodic components. By definition, the periodic-induced component $\Delta y_{p \ell}$ of the resulting error $\Delta y$ is equal to

$$
\Delta y_{p \ell}=\sum_{i} \sum_{j} C_{i j} \cdot A_{\ell i} \cdot \cos \left(\omega_{\ell} \cdot t_{i j}+\varphi_{\ell i}\right)
$$

i.e., to

$$
\Delta y_{p \ell}=\sum_{i} \sum_{j} C_{i j} \cdot A_{\ell i} \cdot\left(\cos \left(\omega_{\ell} \cdot t_{i j}\right) \cdot \cos \left(\varphi_{\ell i}\right)-\sin \left(\omega_{\ell} \cdot t_{i j}\right) \cdot \sin \left(\varphi_{\ell i}\right)\right) .
$$

By combining the terms corresponding to different $j$, we conclude that

$$
\Delta y_{p \ell}=\sum_{i} A_{\ell i} \cdot K_{c i} \cdot \cos \left(\varphi_{\ell i}\right)+\sum_{i} A_{\ell i} \cdot K_{s i} \cdot \sin \left(\varphi_{\ell i}\right),
$$

where $K_{c i} \stackrel{\text { def }}{=} \sum_{j} C_{i j} \cdot \cos \left(\omega_{\ell} \cdot t_{i j}\right)$ and $K_{s i} \stackrel{\text { def }}{=} \sum_{j} C_{i j} \cdot \sin \left(\omega_{\ell} \cdot t_{i j}\right)$.

The values $K_{c i}$ and $K_{s i}$ can be explicitly described. Namely:

- One can easily see that if for some small value $\delta>0$, for this sensor $i$, we take $\Delta x_{i}\left(t_{i j}\right)=\delta \cdot \cos \left(\omega_{\ell} \cdot t_{i j}\right)$ for all $j$, and for all other sensors $i^{\prime}$, we take $\Delta x_{i^{\prime}}\left(t_{i^{\prime} j}\right)=$ 0 , then the resulting increase in $y$ will be exactly equal to $\delta \cdot K_{c i}$.

- Similarly, if for this sensor $i$, we take $\Delta x_{i}\left(t_{i j}\right)=\delta \cdot \sin \left(\omega_{\ell} \cdot t_{i j}\right)$ for all $j$, and for all other sensors $i^{\prime}$, we take $\Delta x_{i^{\prime}}\left(t_{i^{\prime} j}\right)=0$, then the resulting increase in $y$ will be exactly equal to $\delta \cdot K_{s i}$.

Once we have determined the coefficients $K_{c i}$ and $K_{s i}$, we need to describe the probability distribution of the $\operatorname{sum} \Delta y_{p \ell}=\sum_{i} A_{\ell i} \cdot K_{c i} \cdot \cos \left(\varphi_{\ell i}\right)+\sum_{i} A_{\ell i} \cdot K_{s i} \cdot \sin \left(\varphi_{\ell i}\right)$. We assumed that all $\varphi_{i}$ are independent (and uniformly distributed). Thus, for the case of multiple sensors, we can apply the Central Limit Theorem and conclude that the distribution of the sum $\Delta y_{p \ell}$ is close to normal.

In general, normal distribution is uniquely determined by its first two moments: mean and variance (or, equivalently, standard deviation). The mean of each sine and cosine term is 0 , so the mean of the sum $\Delta y_{p \ell}$ is zero as well. Since the terms corresponding to different sensors are independent, the variance of the sum is equal to the sum of the variances of individual terms. For each $i$, the mean of the square

$$
\begin{gathered}
\left(A_{\ell i} \cdot K_{c i} \cdot \cos \left(\varphi_{\ell i}\right)+A_{\ell i} \cdot K_{s i} \cdot \sin \left(\varphi_{\ell i}\right)\right)^{2}= \\
A_{\ell i}^{2} \cdot\left(K_{c i}^{2} \cdot \cos ^{2}\left(\varphi_{\ell i}\right)+K_{s i}^{2} \cdot \sin \left(\varphi_{\ell i}\right)+2 \cdot K_{c i} \cdot K_{s i} \cdot \cos \left(\varphi_{\ell i}\right) \cdot \sin \left(\varphi_{\ell i}\right)\right)
\end{gathered}
$$

is equal to $\frac{1}{2} \cdot A_{\ell i}^{2} \cdot\left(K_{c i}^{2}+K_{s i}^{2}\right)$. Thus, the variance of the sum is equal to

$$
\frac{1}{2} \cdot \sum_{i} A_{\ell i}^{2} \cdot\left(K_{c i}^{2}+K_{s i}^{2}\right)
$$


Each amplitude $A_{\ell i}$ can take any value from 0 to the known bound $P_{\ell i}$. The above expression monotonically increases with $A_{\ell i}$, and thus, it attains its largest value when $A_{\ell i}$ takes the largest value $P_{\ell i}$. Thus, the largest possible value of the variance is equal to $\frac{1}{2} \cdot \sum_{i} P_{\ell i}^{2} \cdot\left(K_{c i}^{2}+K_{s i}^{2}\right)$.

Thus, we arrive at the following algorithm for computing the upper bound $\sigma_{p \ell}$ of the standard deviation of the periodic-induced component $\Delta y_{p \ell}$ on the approximation error $\Delta y$.

Propagating periodic-induced component: algorithm. The upper bound $\sigma_{p \ell}$ on the standard deviation of the periodic-induced component $\Delta y_{p \ell}$ can be obtained as follows:

- First, we apply the algorithm $f$ to the measurement results $\tilde{x}_{i}\left(t_{i j}\right)$ and get the estimate $\widetilde{y}$.

- Then, we select a small value $\delta$ and for each sensor $i$, we do the following:

- for this sensor $i$, we take $x_{i}^{(c i)}\left(t_{i j}\right)=\widetilde{x}_{i}\left(t_{i j}\right)+\delta \cdot \cos \left(\omega_{\ell} \cdot t_{i j}\right)$ for all moments $j$;

- for all other sensors $i^{\prime} \neq i$, we take $x_{i^{\prime}}^{(c i)}\left(t_{i^{\prime} j}\right)=\tilde{x}_{i}\left(t_{i^{\prime} j}\right)$;

- substitute the resulting values $x_{i^{\prime}}^{(c i)}\left(t_{i^{\prime} j}\right)$ into the data processing algorithm $f$ and get the result $y^{(c i)}$;

- then, for this sensor $i$, we take $x_{i}^{(s i)}\left(t_{i j}\right)=\widetilde{x}_{i}\left(t_{i j}\right)+\delta \cdot \sin \left(\omega_{\ell} \cdot t_{i j}\right)$ for all moments $j$;

- for all other sensors $i^{\prime} \neq i$, we take $x_{i^{\prime}}^{(s i)}\left(t_{i^{\prime} j}\right)=\widetilde{x}_{i}\left(t_{i^{\prime} j}\right)$;

- substitute the resulting values $x_{i^{\prime}}^{(s i)}\left(t_{i^{\prime} j}\right)$ into the data processing algorithm $f$ and get the result $y^{(s i)}$.

- Finally, we estimate the desired bound $\sigma_{p \ell}$ as

$$
\sigma_{p \ell}=\sqrt{\frac{1}{2} \cdot \sum_{i} P_{\ell i}^{2} \cdot\left(\left(\frac{y^{(c i)}-\widetilde{y}}{\delta}\right)^{2}+\left(\frac{y^{(s i)}-\widetilde{y}}{\delta}\right)^{2}\right)}
$$

Metrological comment. In some practical situations, instead of the absolute bound $P_{\ell i}$ on the amplitude of the corresponding periodic error components, practitioners often describe relative accuracy $\delta_{\ell i}$ such as $5 \%$ or $10 \%$. In this case, a reasonable way to describe the absolute bound is to determine it as $\sigma_{i}=\delta_{i} \cdot m_{i}$, i.e., by multiplying the given value $\delta_{i}$ and the mean square value of the signal

$$
m_{i}=\sqrt{\frac{1}{T_{i}} \cdot \sum_{j}\left(\widetilde{x}_{i}\left(t_{i j}\right)\right)^{2}} .
$$

Example. To test our algorithm, we have applied it to compute the corresponding error component in the problem of estimating carbon and water fluxes described 
in the paper [14], where such the notion of a periodic error component was first introduced. Our numerical results are comparable with the conclusions of that paper. In the future, we plan to apply all the above algorithms to the results obtained by the sensors on the Jornada Experimental Range Eddy covariance tower and on the nearby robotic tram, and by the affiliated stationary sensors [5, 6, 7, 8, 11, 17].

\section{Conclusion}

In many application areas, it is necessary to process time series. In this processing, it is necessary to take into account uncertainty with which we know the corresponding values. Traditionally, measurement uncertainty has been classified into systematic and random components. However, for time series, this classification is often not sufficient, especially in the analysis of seasonal meteorological and environmental time series. To describe real-life measurement uncertainty more accurately, researchers have come up with a semi-empirical idea of introducing a new type of measurement uncertainty - that corresponds to periodic errors. In this paper, we provide a mathematical justification for this new error component, and describe efficient algorithms for propagating the resulting three-component uncertainty.

Acknowledgements This work was supported in part by the National Science Foundation grants HRD-0734825 (Cyber-ShARE Center of Excellence) and DUE-0926721, and by Grant 1 T36 GM078000-01 from the National Institutes of Health.

The authors are greatly thankful to the anonymous referees for valuable suggestions.

\section{References}

1. Aubinet M., Vesala, T., Papale, D., eds.: Eddy Covariance - A Practical Guide to Measurement and Data Analysis, Springer, Dordrecht, Hiedelberg, London, New York (2012)

2. Cormen, T.H., Leiserson, C.E., Rivest, R.L., Stein, C.: Introduction to Algorithms, MIT Press, Cambridge, Massachusetts (2009)

3. Cryer, J.D., Chan, K.-S.: Time Series Analysis, Springer, New York (2010)

4. Garloff, J.: Zur intervallmässigen Durchführung der schnellen Fourier-Transformation. ZAMM 60, T291-T292 (1980)

5. Herrera, J.: A robotic tram system used for understanding the controls of Carbon, water, of energy land-atmosphere exchange at Jornada Experimental Range, Abstracts of the 18th Symposium of the Jornada Basin Long Term Ecological Research Program, Las Cruces, New Mexico, July 15, 2010 (2010)

6. Jaimes, A.: Net ecosystem exchanges of Carbon, water and energy in creosote vegetation cover in Jornada Experimental Range, Abstracts of the 18th Symposium of the Jornada Basin Long Term Ecological Research Program, Las Cruces, New Mexico, July 15, 2010 (2010)

7. Jaimes, A., Tweedie, C.E., Peters, D.C., Herrera, J., Cody, R.: GIS-tool to optimize site selection for establishing an eddy covariance and robotic tram system at the Jornada Experimental Range, New Mexico, Abstracts of the 18th Symposium of the Jornada Basin Long Term Ecological Research Program, Las Cruces, New Mexico, July 15, 2010 (2010) 
8. Jaimes, A., Tweedie, C.E., Peters, D.C., Ramirez, G., Brady, J., Gamon, J., Herrera, J., Gonzalez, L.: A new site for measuring multi-scale land-atmosphere Carbon, water and energy exchange at the Jornada Experimental Range, Abstracts of the 18th Symposium of the Jornada Basin Long Term Ecological Research Program, Las Cruces, New Mexico, July 15, 2010 (2010)

9. Klir, G.J., Yuan, B.: Fuzzy Sets and Fuzzy Logic. Prentice Hall, Upper Saddle River, New Jersey (1995)

10. Kreinovich, V.: Interval computations and interval-related statistical techniques: tools for estimating uncertainty of the results of data processing and indirect measurements. In: Pavese, F., Forbes, A.B. (eds.): Data Modeling for Metrology and Testing in Measurement Science, pp. 117-145. Birkhauser-Springer, Boston (2009)

11. Laney, C., Cody, R., Gallegos, I., Gamon, J., Gandara, A., Gates, A., Gonzalez, L., Herrera, J., Jaimes, A., Kassan, A., Kreinovich, V., Nebesky, O., Pinheiro da Silva, P., Ramirez, G., Salayandia, L., Tweedie, C.: A cyberinfrastructure for integrating data from an eddy covariance tower, robotic tram system for measuring hyperspectral reflectance, and a network of phenostations and phenocams at a Chihuahuan Desert research site, Abstracts of the FLUXNET and Remote Sensing Open Workshop: Towards Upscaling Flux Information from Towers to the Globe, Berkeley, California, June 7-9, 2011, p. 48 (2011)

12. Lee, X., Massman, W., Law, B.: Handbook of Micrometeorology - A Guide for Surface Flux Measurements. Springer, Berlin, Heidelberg, New York (2011)

13. Liu, G., Kreinovich, V.: Fast convolution and fast Fourier transform under interval and fuzzy uncertainty. Journal of Computer and System Sciences 76(1), 63-76 (2010)

14. Moncrieff, J.B., Malhi, Y., Leuning, R.: The propagation of errors in long-term measurements of land-atmospheric fluxes of carbon and water. Global Change Biology 2, 231-240 (1996)

15. Nguyen, H.T., Walker, E.A.: First Course In Fuzzy Logic. CRC Press, Boca Raton, Florida (2006)

16. Rabinovich, S.: Measurement Errors and Uncertainties: Theory and Practice. American Institute of Physics, New York (2005)

17. Ramirez, G.: Quality data in light sensor network in Jornada Experimental Range, Abstracts of the 18th Symposium of the Jornada Basin Long Term Ecological Research Program, Las Cruces, New Mexico, July 15, 2010 (2010)

18. Richardson, A.D., et al.: Uncertainty quanitication. In: Aubinet M., Vesala, T., Papale, D., eds.: Eddy Covariance - A Practical Guide to Measurement and Data Analysis, Springer, Dordrecht, Hiedelberg, London, New York, 173-209 (2012)

19. Sheskin, D.J.: Handbook of Parametric and Nonparametric Statistical Procedures. Chapman and Hall/CRC Press, Boca Raton, Florida (2011)

20. Shumway, R.H., Stoffer, D.S.: Time Series Analysis and Its Applications, Springer, New York (2010) 